\title{
Factors Impacting Stone-Free Rate After Retrograde Intrarenal Surgery for Calyceal Diverticular Calculi
}

This article was published in the following Dove Press journal: Research and Reports in Urology

\section{Chatporn Boonyapalanant \\ Pat Saksirisampant (D) \\ Tawatchai Taweemonkongsap \\ Sunai Leewansangtong \\ Sittiporn Srinualnad \\ Ekkarin Chotikawanich}

Division of Urology, Department of Surgery, Faculty of Medicine, Siriraj Hospital, Mahidol University, Bangkok, Thailand
Correspondence: Ekkarin Chotikawanich Division of Urology, Department of Surgery, Faculty of Medicine, Siriraj Hospital, Mahidol University, Bangkok, Thailand

Tel +66-2-419-8010

Email ekkarinc@yahoo.com
Objective: To evaluate the outcomes of retrograde intrarenal surgery (RIRS) treatment of calyceal diverticular calculi and identify the associated factors affecting post-operative stonefree rate.

Materials and Methods: From August 2015 to May 2019, data of 32 patients with calyceal diverticular calculi who were treated by RIRS in a Siriraj Hospital were retrospectively studied. All operations were performed by the same surgeon using flexible ureterorenoscopy (f-URS) and holmium YAG laser lithotripsy. Calyceal diverticula were identified by our refluxing technique and from the collected demographic, diverticular and stone data. Operative outcomes were retrospectively evaluated. Data were analysed to identify the factors associated with stone-free outcomes. Stone-free was defined as no residual stones remaining after surgery.

Results: Mean age of the patients was 55.7 years. Stone locations were non-lower pole in $81.2 \%$ of cases and lower pole for the remaining $18.8 \%$ of cases. Median stone size was $1.2 \mathrm{~cm}$ with three as the median number of stones per patient. Calcium oxalate was the most common stone composition $(56.3 \%)$. Positions of the diverticulum were anterior calyx $(34.4 \%)$ and posterior calyx (50\%), while the remainder were undetermined (incomplete data). Average length of the diverticular neck was $0.4 \mathrm{~cm}$. Mean operative time was 46 minutes and mean hospital stay was 2.9 days. Complications included fever in three patients $(9.3 \%)$ and sepsis in two patients $(6.3 \%)$, with overall post-operative stone-free rate at $75 \%$. Factors significantly affecting stone-free status were stone size $(P=0.003)$ and length of diverticular neck $(P=0.038)$. Multivariate analysis determined that only stone size had a statistically significant effect on post-operative stone-free status $(P=0.015)$. Cut off point for stone size that increased the chances of a post-operative stone-free outcome was less than $1.5 \mathrm{~cm}$, as determined by the ROC curve.

Conclusion: RIRS was found to be an effective and safe treatment option for the removal of calyceal diverticular calculi. Stone size of less than $1.5 \mathrm{~cm}$ offered a better chance of postoperative stone-free condition.

Keywords: calyceal diverticular calculi, retrograde intrarenal surgery, endoscopy

\section{Introduction}

A calyceal diverticulum is a urothelium-lined cystic dilatation of the renal collecting system. This presents as an uncommon anatomic abnormality of the kidney with incidence of about 4.5 per 1000 population. ${ }^{1}$ However, $9.5-50 \%$ of calyceal diverticula are associated with internal calculi formation that appear to be caused by anatomical obstruction and urine stasis. ${ }^{1-3}$ The disease occurs more commonly in 
women than in men. ${ }^{2,3}$ Nowadays, the treatment option with the optimal post-operative stone-free rate is percutaneous nephrolithotomy (PCNL), ${ }^{4,5}$ whereas retrograde intrarenal surgery (RIRS) is an optional treatment for small calculi located in the upper and middle calyces. ${ }^{5}$

Recently, stone management using the endourological technique has become more advanced, thus RIRS can now treat large calyceal diverticular calculi in the lower calyx with improved treatment outcomes. Previous studies determined that the stone-free rate of treating calyceal diverticular calculi by RIRS was $85 \%{ }^{6}$ However, to the best of our knowledge, there are no published reports concerning the factors that affect the post-operative stone-free rate for RIRS.

Therefore, this study was conducted to identify factors affecting treatment outcomes of calyceal diverticular calculi by RIRS.

\section{Materials and Methods}

This study was approved by the Ethics Committee of the Faculty of Medicine Siriraj Hospital, Mahidol University, Thailand, protocol number 738/2560(EC3). In this IRB certificate of approval for the study, patient consent was waived for this retrospective study due to a retrospective study protocol in Siriraj Hospital. However, all patient data retained confidentiality and compliance with the Declaration of Helsinki. Charts of patients with renal diverticular calculi who underwent RIRS at Siriraj Hospital from August 2015 to May 2019 were retrospectively reviewed. All surgical procedures were performed by the same experienced urologist. Each patient was diagnosed before the operation by a computerized tomography (CT) scan or intravenous pyelography (IVP) to evaluate the calculi and calyceal diverticulum. RIRS was considered to be the first option for all cases.
Under general anesthesia, all patients were treated using flexible ureterorenoscopy (f-URS) and holmium YAG laser lithotripsy. Enlarging the opening of the calyceal diverticulum was also performed by laser before lithotripsy was conducted to remove the stone fragments. The location of the diverticulum was confirmed before using a laser to widen its opening by the refluxing technique comprising the contrast medium methylene blue. The mixture was injected through the ureteroscope in front of the suspected diverticular opening, previously confirmed by fluoroscopy, until filling the entire collecting system. Endoscopy with normal saline was continued until the methyl blue was cleared and fluid irrigation was then discontinued. Refluxing of the methylene blue was revealed from the diverticular opening (Figure 1). This technique is useful to identify the opening before precise cutting using a laser. The opening and the neck were cut until the ureteroscope was able to enter into the diverticulum. Laser lithotripsy was performed following a similar technique for non-diverticular calculi depending on the size of the stone. At the end of the procedure, a contrast study was performed to evaluate the diameter of the diverticular neck and confirm that there was no leakage. A double-J stent was retained while the upper coil was inside the diverticulum (Figure 2). The stent was removed at the first follow-up, 2 weeks after the operation. Ultrasonography or KUB film was performed to determine the stone status by radiologists as a matter of routine. KUB film was used to evaluate opaque stones, while ultrasonography was used to evaluate nonopaque stones. Stone-free status described no postoperative residual stones.

Demographic, diverticular, and stone data were collected. All patients were categorized into two groups as stone-free and remaining with residual stones. Data from
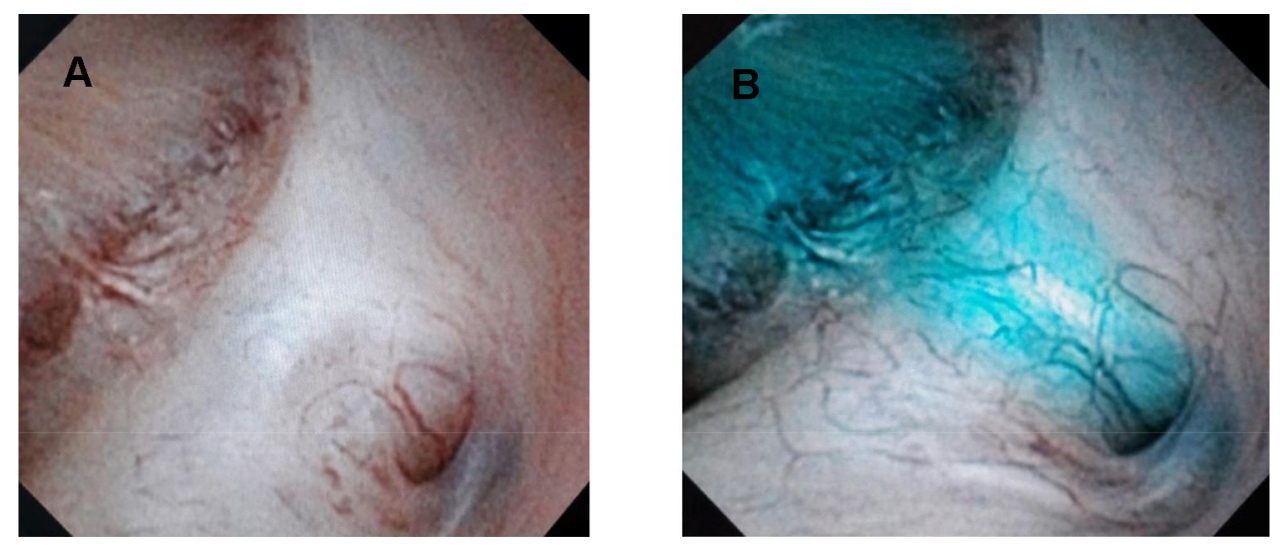

Figure I A refluxing technique with methylene blue was used to identify the diverticular opening. (A) Suspected opening. (B) Refluxing of methylene blue through the opening. 

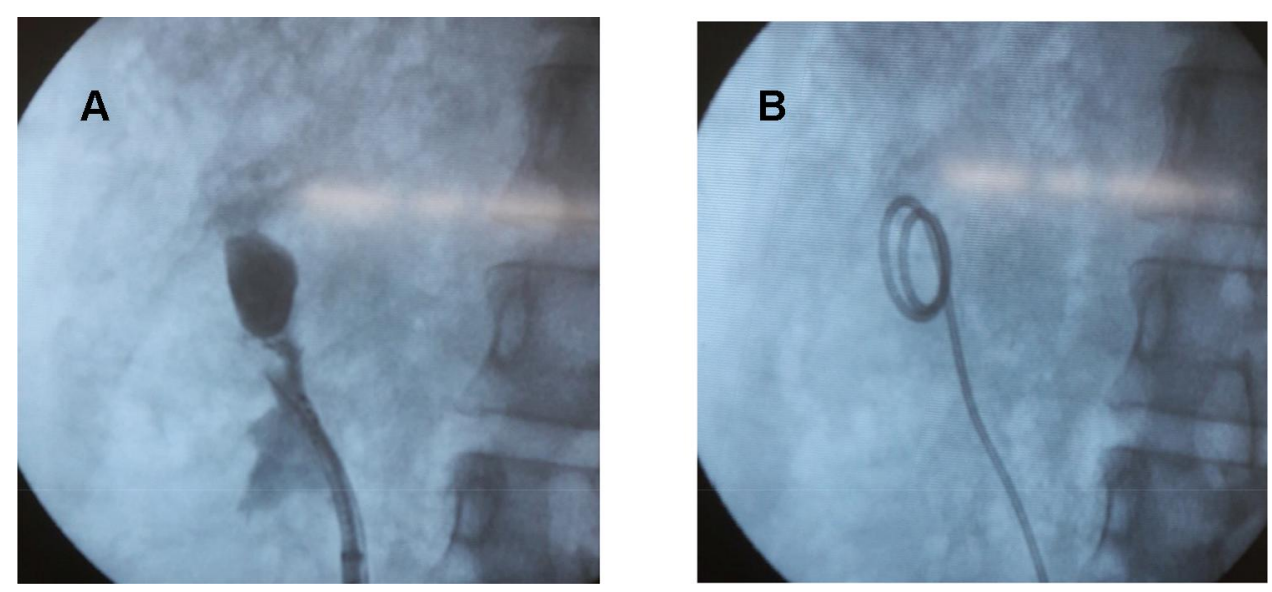

Figure 2 At the end of the procedure, a contrast study was performed and a double-J stent was retained inside the diverticulum. (A) Diverticular neck after widening. (B) Upper coil of double-J stent was in the diverticulum.

both groups were analyzed to identify factors associated with stone-free outcomes.

Statistical analysis was conducted using PASW (SPSS) Statistics version 18.0 software. Demographic data were presented as mean $\pm \mathrm{SD}$. In the case of a normal distribution, independent sample $t$-test, Fisher's exact test, and Pearson's chi-square were used to compare the results between both groups using a $P$-value of less than 0.05 to determine statistical significance. An ROC curve was used to determine the cut-off point of stone size and length of diverticular neck on post-operative stone-free outcome. Logistic regression for multivariate analysis was used to identify the statistically significant factors that affected the stone-free rate.

\section{Results}

Thirty-two patients were enrolled in this study, with demographic data shown in Table 1. Ten patients (31.3\%) were male and $22(68.8 \%)$ were female. Mean age was 55.7 years old and the most common presenting symptom was pyuria.

Stone location was non-lower pole in $81.2 \%$ of cases and lower pole for the remaining $18.8 \%$ of cases. Overall median stone size was $1.2 \mathrm{~cm}$, with the median number of stones per patient being three. The most common stone composition was calcium oxalate $(56.3 \%)$, followed by calcium phosphate (25\%). Positions of calyceal diverticulum were $34.4 \%$ anterior, $50.0 \%$ posterior, and $15.6 \%$ unknown (incomplete data). Stones located in the anterior and posterior diverticulum had median cumulative sizes of $1.21 .6 \mathrm{~cm}$ respectively, while the average length of the diverticular neck was $0.4 \mathrm{~cm}$.
Mean operative time was 46 minutes and mean hospital stay was 2.9 days, with an average follow-up time of 2.3 weeks. Incidence of complications after surgery was $15.6 \%$ (Table 2). Three patients $(9.3 \%)$ had postoperative fever and two patients $(6.3 \%)$ developed sepsis. All these patients were successfully treated with

Table I Demographic Data

\begin{tabular}{|l|l|}
\hline Characteristics & Numbers \\
\hline $\begin{array}{r}\text { Sex } \\
\bullet \quad \text { Male; } n \text { (\%) }\end{array}$ & $10(31.3)$ \\
$22(68.8)$
\end{tabular}


Table 2 Complications

\begin{tabular}{|l|l|}
\hline Complication & Incidence \\
\hline Fever; $\mathbf{n}(\%)$ & $3(9.3)$ \\
Sepsis; $\mathbf{n}(\%)$ & $2(6.3)$ \\
\hline
\end{tabular}

antibiotics. There was no major ureter injury related to f-URS, and the stone-free rate after the operation was $75 \%$.

Factors affecting stone-free status included stone size, stone position, position of diverticulum, and length of diverticular neck, measured from the opening to the diverticulum (Figure 3). Stone size and diverticular neck length were evaluated utilizing the ROC curve to determine the optimal cut-off point. For stone size, this was $1.5 \mathrm{~cm}$ with sensitivity of $75 \%$ and specificity of $87.5 \%$ (Figure 4 ), while the optimal length of the diverticular neck was $0.4 \mathrm{~cm}$ with sensitivity and specificity of $70.875 \%$ (Figure 5). These factors were compared between the stone-free group and the residual stone group, with results shown in Table 3. There were statistically significant differences for stone size $(P$-value $=0.003)$ and length of diverticular neck $(P$-value $=0.038)$, while no statistically significant differences were recorded for stone location $(P$-value $=0.625)$ and position of diverticulum $(P$-value $=1.000)$. However, multivariate analysis revealed that only stone size was statistically significant $(P$-value $=0.015)$, as shown in Table 4.

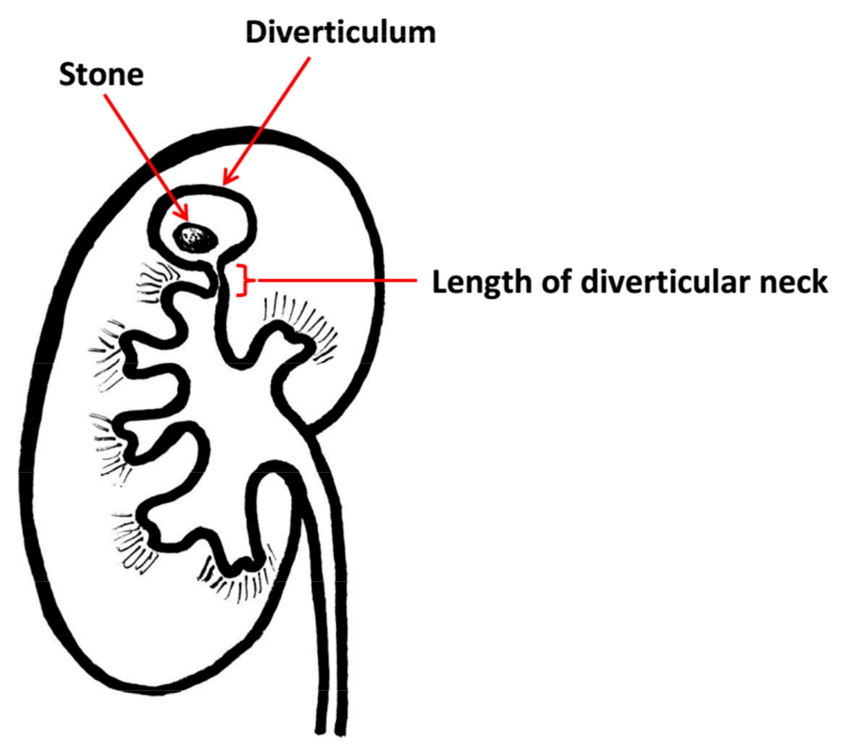

Figure 3 The length of the diverticular neck was measured from the opening to the diverticulum.

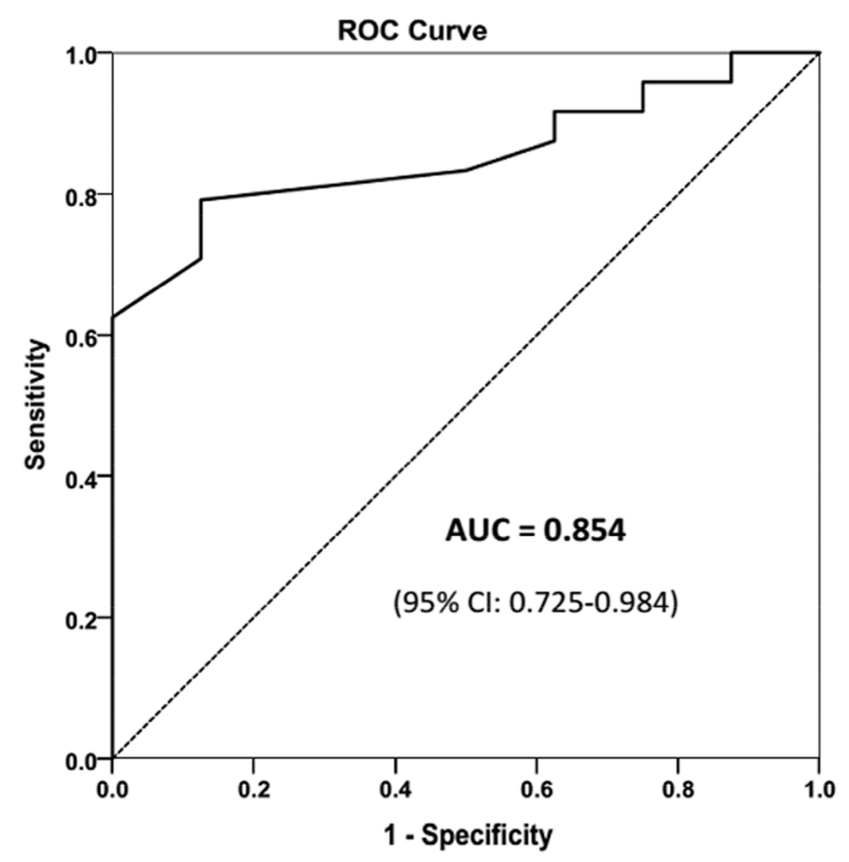

Figure 4 ROC curve, optimal cut-off point of stone size.

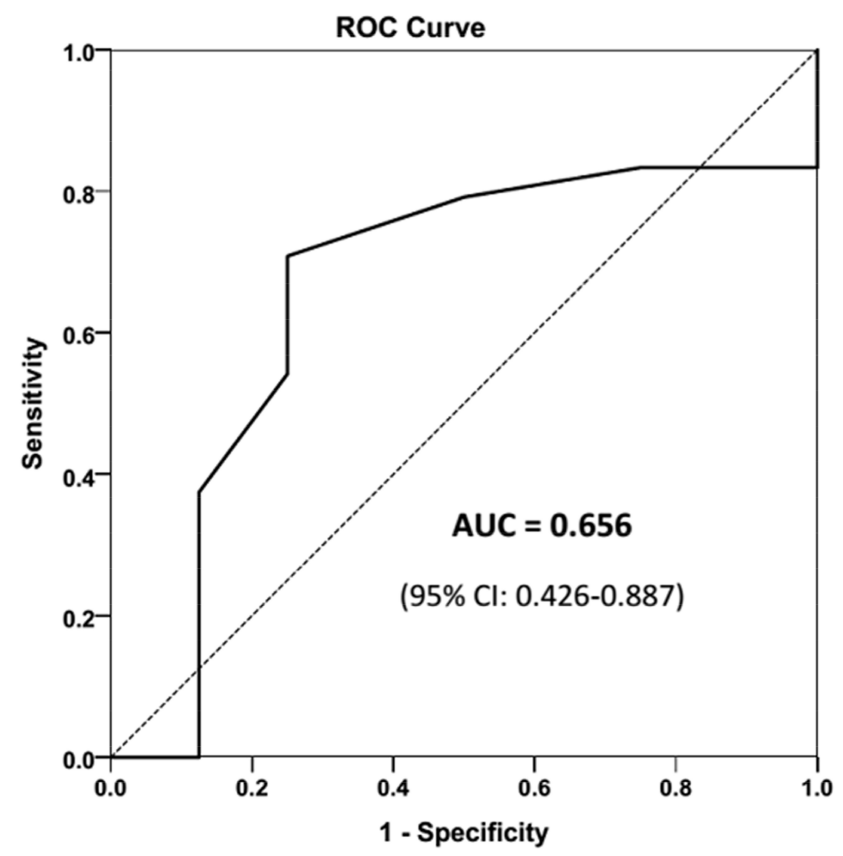

Figure 5 ROC curve, optimal cut-off point of diverticulum neck length.

\section{Discussion}

In recent years, PCNL has been accepted as the first-line treatment of calyceal diverticular calculi, while RIRS was an optional procedure for small stones located in the upper or middle calyx. Bas et $\mathrm{al}^{7}$ reported that the overall stone-free rate for PCNL was $82.8 \%$, with $62 \%$ of stones in the lower calyx, while the overall stone-free rate for URS was $76 \%$, 
Table 3 Outcomes

\begin{tabular}{|c|c|c|c|}
\hline & Stone Free & Residual Stone & $P$-value \\
\hline $\begin{array}{l}\text { Stone position } \\
\text { - Non-lower pole; n (\%) } \\
\text { - Lower pole; n (\%) }\end{array}$ & $\begin{array}{l}20(76.9) \\
4(66.7)\end{array}$ & $\begin{array}{l}6(23.1) \\
2(33.3)\end{array}$ & 0.625 \\
\hline $\begin{array}{l}\text { Stone size } \\
-\quad<1.5 \mathrm{~cm} ; \mathrm{n}(\%) \\
-\quad \geq 1.5 \mathrm{~cm} \text {; n (\%) }\end{array}$ & $\begin{array}{l}18(94.7) \\
6(46.2)\end{array}$ & $\begin{array}{l}\text { I (5.3) } \\
7(53.8)\end{array}$ & 0.003 \\
\hline $\begin{array}{l}\text { Diverticulum position } \\
\text { - Anterior; n (\%) } \\
\text { - Posterior; n (\%) }\end{array}$ & $\begin{array}{l}8(72.7) \\
12(75.0)\end{array}$ & $\begin{array}{l}3(27.3) \\
4(25.0)\end{array}$ & 1.000 \\
\hline $\begin{array}{l}\text { Diverticular neck length } \\
-\quad<0.4 \mathrm{~cm} ; \mathrm{n}(\%) \\
-\quad \geq 0.4 \mathrm{~cm} ; \mathrm{n}(\%)\end{array}$ & $\begin{array}{l}17(89.5) \\
7(53.8)\end{array}$ & $\begin{array}{l}2(10.5) \\
6(46.2)\end{array}$ & 0.038 \\
\hline
\end{tabular}

Table 4 Multivariate Analysis of the Factors Affecting the StoneFree Rate

\begin{tabular}{|c|c|c|c|}
\hline & Adjusted OR & $95 \% \mathrm{Cl}$ & $P$-value \\
\hline $\begin{array}{l}\text { Stone size } \\
\bullet \quad \geq 1.5 \mathrm{~cm} \\
-\quad<1.5 \mathrm{~cm}\end{array}$ & $\begin{array}{l}\text { I (ref) } \\
21.00\end{array}$ & $(1.82-242.32)$ & 0.015 \\
\hline $\begin{array}{l}\text { Diverticular neck length } \\
-\quad \geq 0.4 \mathrm{~cm} \\
-\quad<0.4 \mathrm{~cm}\end{array}$ & $\begin{array}{l}\text { I (ref) } \\
7.29\end{array}$ & $(0.83-63.79)$ & 0.073 \\
\hline
\end{tabular}

Abbreviations: $\mathrm{OR}$, odds ratio; $\mathrm{Cl}$, confidence interval; ref, reference.

with $80 \%$ of stones in the non-lower calyx. In our study, total stone-free rate was $75 \%$, which included $18.8 \%$ of lower pole calculi. There was no patient selection and all of the diverticular calculi patients underwent RIRS as the first treatment, including both favorable and unfavorable outcomes. However, post-operative stone-free rates were $94.7 \%$ for stones with a diameter less than $1.5 \mathrm{~cm}$ and $89.5 \%$ for stones located in the diverticulum, with a diverticular neck length of less than $0.4 \mathrm{~cm}$, regardless of the calyceal location. The diverticular neck length was considered to be a factor affecting stone-free status, as a longer neck length caused misdirection in laser cutting from the opening to the diverticulum. However, a stone diameter of less than $1.5 \mathrm{~cm}$ was the only significant factor affecting stone-free rates according to multivariate analysis.

Previous studies reported that an anteriorly located diverticulum is difficult to manage by PCNL due to the longer distance or inaccessible angle. ${ }^{1,8}$ Significant hemorrhage is one of the complications when the tract has to traverse through the renal parenchyma to access anterior lesions. ${ }^{8,9}$ Percutaneous access is commonly preferred for a posterior diverticulum as a direct puncture to reach the stone. ${ }^{7,10}$ In this study, $34.4 \%$ of cases had the diverticulum located in the anterior position, with stones at a median size of $1.2 \mathrm{~cm}$. The success rate for anterior diverticula was $72.7 \%$ stone-free, although this was not statistically different from the posterior case. Thus, it was worthwhile to consider RIRS as a procedure for diverticular calculi located in the anterior calyx.

After stone removal, destroying the diverticular mucosa to prevent recurrent stasis of urine in the diverticulum was recommended, especially for percutaneous access. For retrograde access, after widening the diverticular neck, urine was able to pass into the calyx as an internal shunt; therefore, here, the mucosa of the diverticulum were not destroyed. A ureteral stent was retained in all cases. The upper coil of the stent was located in the diverticulum to drain the urine, while the stent passed through the diverticular neck to prevent stenosis. The mean follow-up period in this study was 2.3 weeks with no diverticulum recurrence. However, a longer follow-up is recommended to ensure no further diverticulum recurrence.

The occurrence of complications in this study was similar to levels reported in previous studies ${ }^{5,11-13}$ at $15.6 \%$. The most common complication was fever $(9.3 \%)$ followed by sepsis $(6.3 \%)$. These were successfully treated by antibiotics without further intervention being required. The rate of infectious complications in this study was comparable to that of RIRS for kidney stones without diverticulum. ${ }^{14,15}$

Our results indicated that RIRS achieved a $94.7 \%$ postoperative stone-free result for stones less than $1.5 \mathrm{~cm}$ in diameter, regardless of diverticulum position.

There were some limitations in this study. These included bias, which is inevitable in a retrospective research study, incomplete data, and a small number of patients due to low incidence of the disease.

\section{Conclusions}

RIRS is a treatment option for treating calyceal diverticular calculi. Stone size less than $1.5 \mathrm{~cm}$ was a factor in achieving optimal post-operative stone-free rate. A diverticular neck length of less than $0.4 \mathrm{~cm}$ had a higher chance of attaining a stone-free result. Operative outcomes for the anterior and posterior calyceal diverticulum were similar.

\section{Abbreviations}

RIRS, retrograde intrarenal surgery; KUB, kidneys, ureters, and bladder; CT, computerized tomography; IVP, 
intravenous pyelogram; US, ultrasound; PCNL, percutaneous nephrolithotomy; URS, ureteroscopy; f-URS, flexible ureteroscopy; ROC, receiver operating characteristics; AUC, area under the curve.

\section{Disclosure}

The authors report no conflicts of interest in this work.

\section{References}

1. Gross AJ, Herrmann RW. Management of stones in calyceal diverticulum. Curr Opin Urol. 2007;17:136-140. doi:10.1097/ MOU.0b013e328011bcd3

2. Timmons JW Jr, Malek RS, Hattery RR, Deweerd JH. Caliceal diverticulum. $J$ Urol. 1975;114:6-9. doi:10.1016/S0022-5347(17) 66930-1

3. Middleton AW Jr, Pfister RC. Stone-containing pyelocaliceal diverticulum: embryogenic, anatomic, radiologic and clinical characteristics. J Urol. 1974;111:2-6. doi:10.1016/S0022-5347(17)59872-9

4. Auge BK, Munver R, Kourambas J, Newman GE, Preminger GM. Endoscopic management of symptomatic caliceal diverticula: a retrospective comparison of percutaneous nephrolithotripsy and ureteroscopy. J Endourol. 2002;16:557-563. doi:10.1089/08927790 2320913233

5. Waingankar N, Hayek S, Smith AD, Okeke Z. Calyceal diverticula: a comprehensive review. Rev Urol. 2014;16:29-43.

6. Erkurt B, Kiremit MC, Altay B, et al. Is retrograde flexible nephrolithotripsy feasible for calyceal diverticular stone? Urolithiasis. 2014;42:347-351. doi:10.1007/s00240-014-0672-1
7. Bas O, Ozyuvali E, Aydogmus Y, et al. Management of calyceal diverticular calculi: a comparison of percutaneous nephrolithotomy and flexible ureterorenoscope. Urolithiasis. 2015;43:155-161. doi:10.1007/s00240-014-0725-5

8. Matlaga BR, Jansen JP, Meckley LM, Byrne TW, Lingeman JE. Treatment of ureteral and renal stones: a systematic review and meta-analysis of randomized, controlled trials. $J$ Urol. 2012;188:130-137. doi:10.1016/j.juro.2012.02.2569

9. Tepeler A, Bozkurt OF, Resorlu B, et al. Is the percutaneous nephrolithotomy procedure complicated in patients with anterior caliceal stones? Urol Int. 2013;90:389-393. doi:10.1159/000345711

10. Canales B, Monga M. Surgical management of the calyceal diverticulum. Curr Opin Urol. 2003;13:255-260. doi:10.1097/ 00042307-200305000-00015

11. Chong TW, Bui MH, Fuchs GJ. Calyceal diverticula. Ureteroscopic Management Urol Clin North Am. 2000;27:647-654. doi:10.1016/ S0094-0143(05)70114-2

12. Zhang JQ, Wang Y, Zhang JH, Zhang XD, Xing NZ. Retrospective analysis of ultrasound-guided flexible ureteroscopy in the management of calyceal diverticular calculi. Chin Med J. 2016;129:2067-2073. doi:10.4103/0366-6999.189060

13. Grasso M, Lang G, Loisides P, Bagley D, Taylor F. Endoscopic management of the symptomatic caliceal diverticular calculus. J Urol. 1995;153:1878-1881. doi:10.1016/S0022-5347(01)67337-3

14. Li T, Sun XZ, Lai DH, Li X, He YZ. Fever and systemic inflammatory response syndrome after retrograde intrarenal surgery: risk factors and predictive model. Kaohsiung J Med Sci. 2018;34:400-408. doi:10.1016/j.kjms.2018.01.002

15. Baseskioglu B. The prevalence of urinary tract infection following flexible ureterenoscopy and the associated risk factors. Urol J. 2019;16:439-442. doi:10.22037/uj.v0i0.4340
Research and Reports in Urology

\section{Publish your work in this journal}

Research and Reports in Urology is an international, peer-reviewed, open access journal publishing original research, reports, editorials, reviews and commentaries on all aspects of adult and pediatric urology in the clinic and laboratory including the following topics: Pathology, pathophysiology of urological disease; Investigation and treatment of urological disease; Pharmacology of drugs used for the treatment of urological disease. The manuscript management system is completely online and includes a very quick and fair peer-review system, which is all easy to use. Visit http://www.dovepress.com/ testimonials.php to read real quotes from published authors. 